Pacific Journal of Mathematics

NONLINEAR RELATIONSHIPS BETWEEN OSCILLATION AND 


\title{
NONLINEAR RELATIONSHIPS BETWEEN OSCILLATION AND ASYMPTOTIC BEHAVIOR
}

\author{
Allan Edelson aNd KURT Kreith
}

\begin{abstract}
This paper establishes various relationships between oscillation and asymptotic behavior of nonlinear differentional equations of the form $y^{(2 n)}-f(t, y)=0$. Its results constitute a generalization of similar relationships known to hold for linear fourth order equations.
\end{abstract}

For the fourth order differential equation

$$
y^{i v}-p(t) y=0,
$$

with $p(t)$ positive and continuous in $[0, \infty)$, it is well known [1] that (1.1) always possesses nonoscillatory solutions of the following two types:

(i ) $y, y^{\prime}, y^{\prime \prime}, y^{\prime \prime \prime}$ are eventually positive;

(ii) $y,-y^{\prime}, y^{\prime \prime},-y^{\prime \prime \prime}$ are eventually positive.

We say that (1.1) satisfies condition $(H)$ if every eventually positive solution is of type (i) or (ii) above. Furthermore, we say that (1.1) is oscillatory (0) if it has a nontrivial solution with arbitrarily large zeros. Ahmad [1] has shown that (1.1) is oscillatory if and only if it satisfies condition $(\mathrm{H})$-i.e., that $(0) \Leftrightarrow(\mathrm{H})$.

In considering the relationship between oscillation and asymptotic behavior of nonlinear equations of the the form

$$
y^{(2 n)}-f(t, y)=0,
$$

it will be assumed that $f(t, y)$ is continuous, satisfies

$$
\operatorname{sgn} f(t, y)=\operatorname{sgn} y
$$

in $[0, \infty) \times(-\infty, \infty)$, and that $f(t, y)$ is sufficiently regular so that solutions of the initial value problem associated with (1.2) exist and vary continuously with initial data in $\left[t_{0}, \infty\right)$ for any initial point $t_{0} \geqq 0$. Under these assumptions it can be shown [6] that (1.2) always possesses solutions of the following types:

(i) there exists a $t_{0} \geqq 0$ such that $y^{(j)}(t)$ is positive for all $t>t_{0}$ and $0 \leqq j \leqq 2 n-1$, in which case we say that $y(t)$ is positively strongly increasing (p.s.i.) on $\left(t_{0}, \infty\right)$.

(ii) there exists a $t_{0} \geqq 0$ such that $(-1)^{j} y^{(j)}(t)$ is positive for all $t>t_{0}$ and $0 \leqq j \leqq 2 n-1$, in which case we say that $y(t)$ is positively strongly decreasing (p.s.d.) on $\left(t_{0}, \infty\right)$. We shall also have occasion to refer to negatively strongly decreasing (n.s.d.) and negatively 
strongly increasing (n.s.i.) solutions which are analogously defined by the condition $y^{(j)}(t)$ is eventually negative and $(-1)^{j} y^{(j)}(t)$ is eventually negative for $0 \leqq j \leqq 2 n-1$. In the linear case $y(t)$ is p.s.i. [p.s.d.] on $\left(t_{0}, \infty\right)$ if and only if $-y(t)$ is n.s.d. [n.s.i.] on $\left(t_{0}, \infty\right)$. With the above terminology it is natural to say that (1.2) satisfies condition (H) if every eventually positive solution is either p.s.i. or p.s.d. and every eventually negative solution is either n.s.d. or n.s.i. Condition (0) is again said to be satisfied if (1.2) possesses an oscillatory solution.

In $\S 2$ below we show that the implication $(\mathrm{H}) \Rightarrow(0)$ holds for the equation (1.2). This generalizes a related result by Lovelady [8] for the linear equation

$$
y^{(2 n)}-p(t) y=0 .
$$

The more difficult implication $(0) \Rightarrow(\mathrm{H})$ is considered in $\S 3$ for the linear equation (1.4). Here we observe that some recent results of Elias [2] generalize Ahmad's results if condition (0) is reformulated in terms of conjugate or focal points rather than the existence of an oscillatory solution. Such a reformulation is equivalent to (0) in the case $n=2$. In $\S 4$ we consider nonlinear forms of the implication $(0) \Rightarrow(\mathrm{H})$ for the equation (1.3) in case $n=2$. Writing (1.3) as a system

$$
\frac{d^{2} y}{d t^{2}}=x ; \frac{d^{2} x}{d t^{2}}=f(t, y)
$$

we interpret solutions of (1.3) as trajectories in the $(x, y)$-plane. Our generalizations of Ahmad's result consist of showing the incompatibility of trajectories corresponding to arbitrarily large focal points with the existence of asymptotic trajectories which violate condition $(\mathrm{H})$.

2. Oscillation criteria. For the nonlinear equation

$$
y^{(2 n)}-f(t, y)=0 \quad 0 \leqq t<\infty
$$

satisfying (1.3) and the other conditions set forth in $\S 1$, it follows readily that any nontrivial solution satisfying $y^{(j)}\left(t_{0}\right) \geqq 0$ [@0] for $0 \leqq j \leqq 2 n-1$ is p.s.i. [n.s.d.] on $\left(t_{0}, \infty\right)$. We also have the following.

Lemma 2.1. If $y(t)$ is a solution of $(2.1)$ on $(0, \infty)$ which is p.s.d. [n.s.i.] on $\left(t_{0}, \infty\right)$ for some $t_{0}>0$, then $y(t)$ is p.s.d. [n.s.i.] on $(0, \infty)$.

Proof. Rewriting (2.1) as a vector system 


$$
\underline{x}^{\prime \prime}=\underline{f}(t, \underline{x})
$$

with $\underline{x}=\operatorname{col}\left(x_{1}, \cdots, x_{n}\right), x_{i+1}=y^{(2 i)}$ for $0 \leqq i \leqq n-1$, and $\underline{f}=$ $\operatorname{col}\left(x_{2}, \cdots, x_{n}, f\left(t, x_{1}\right)\right)$, we use the notation $\underline{x}>\underline{0}[\geqq \underline{0}]$ in case $x_{i}>0$ $[\geqq 0]$ for all $i, 1 \leqq i \leqq n$. A solution of (2.1) which is p.s.d. on $\left(t_{0}, \infty\right)$ may then be intepreted as a trajectory $\Gamma(t)$ lying in the positive $n$-tant

$$
I^{+}=\left\{\underline{x} \mid x_{i}>0 \text { for } 1 \leqq i \leqq n\right\}
$$

and satisfying $\underline{x}^{\prime}(t)<\underline{0}$ for $t_{0}<t<\infty$. If $y(t)$ is p.s.d. on $\left(t_{0}, \infty\right)$ but not on $(0, \infty)$ then there exists a maximal interval $(\tilde{t}, \infty)$ on which $y(t)$ is p.s.d., and we would have $x_{i_{0}}^{\prime}(\widetilde{t}) \geqq 0$ for some $i_{0}$. However, since $x_{i_{0}}^{\prime \prime}(t)>0$ for all $t>\widetilde{t}$ we would have $x_{i_{0}}^{\prime}(t)>0$ on $\left(t_{0}, \infty\right)$, contradicting the assmption that $y(t)$ is p.s.d. A similar argument deals with the case of solutions which are n.s.i. on $\left(t_{0}, \infty\right)$.

Continuing in the geometric setting suggested by the proof of Lemma 2.1, we represent the initial value problem corresponding to (2.1) in the system form

$$
\begin{aligned}
& \underline{x}^{\prime \prime}=f(t, \underline{x}) ; t_{0} \leqq t<\infty \\
& \underline{x}\left(t_{0}\right)=\underline{x}^{0} ; \underline{x}^{\prime}\left(t_{0}\right)=\underline{x}^{1} .
\end{aligned}
$$

A solution $\underline{x}(t)$ of $(2.3)$ is then determined by the initial data $\left(\underline{x}^{0}, \underline{x}^{1}\right)$ in $\boldsymbol{R}^{2 n}$. We introduce the additional notation

$$
\begin{aligned}
& I^{-}=\left\{\underline{x} \mid x_{i}<0 \text { for } 1 \leqq i \leqq n\right\} \\
& I^{++}=I^{+} \times I^{+} ; I^{+-}=I^{+} \times I^{-} ; \text {etc } .
\end{aligned}
$$

so that a solution $y(t)$ of $(2.1)$ is p.s.i. on $\left(t_{0}, \infty\right)$ if and only if $\left(\underline{x}(t), \underline{x}^{\prime}(t)\right) \in I^{++}$for $t_{0}<t<\infty$, while $y(t)$ is p.s.d. on $\left(t_{0}, \infty\right)$ if and only if $\left(\underline{x}(t), \underline{x}^{\prime}(t)\right) \in I^{+-}$for $t_{0}<t<\infty$, etc.

THEOREM 2.2. If (2.1) satisfies condition (H), then it has oscillatory solutions.

Proof. Fix $t_{0} \in[0, \infty)$ and let [p.s.i.] denote the set of initial data $\left(\underline{x}^{0}, \underline{x}^{1}\right) \in R^{2 n}$ for which the corresponding solution $\underline{x}(t)$ is eventually p.s.i. The continuous dependence of solutions on initial data implies that [p.s.i.] is an open subset of $\boldsymbol{R}^{2 n}$ containing $I^{++}$; analogously [n.s.d.] is an open subset of $\boldsymbol{R}^{2 n}$ containing $I^{--}$. On the other hand, by Lemma 2.1 [p.s.d.] and [n.s.i.] are subsets of $I^{+-}$ and $I^{-+}$respectively. In order to prove the existence of oscillatory solutions it suffices to show [p.s.i.] $\cup[$ n.s.d.] $\cup[$ p.s.d.] $\cup[$ n.s.i.] is a proper subset of $\boldsymbol{R}^{2 n}-\{0\}$-i.e., that for $n>1$ [p.s.d.] $\cup$ [n.s.i.] cannot effect a separation of the open subsets [p.s.i.] and [n.s.d.] in $\boldsymbol{R}^{2 n}-$ 
\{0\}. Since [p.s.d.] $\subset I^{+-}$and [n.s.i.] $\subset I^{-+}$, it clearly suffices to show that $I^{+-} \cup I^{-+}$cannot effect a separation of the open sets [p.s.i.] and [n.s.d.]. Let $\underline{u}=(1, \cdots, 1) \in R^{n}$, and $\underline{h}=(\underline{u},-\underline{u}) \in R^{2 n}$. The hyperplane orthogonal to $\underline{h}$ in $R^{2 n}$ can be described by $H=$ $\left\{\left(\underline{X}^{0}, \underline{X}^{1}\right)\left|<\left(\underline{X}^{0}, \underline{X}^{1}\right),(\underline{u},-\underline{u})\right\rangle=0\right\}$. Then $H_{0}=H-\{(0,0)\}$ is homeomorphic to $R^{2 n-1}-\{0\}$, and for $n>1$ is connected. It is clear that $H_{0} \cap\left(I^{+-} \cup I^{-+}\right)=\varnothing$. If [p.s.i.] $\cup\left[\right.$ n.s.d.] $\cup\left[\right.$ p.s.d.] $\cup\left[\right.$ n.s.i.] $=\boldsymbol{R}^{2 n}-$ $\{0\}$, then $H_{0}=\left(H_{0} \cap\right.$ p.s.i.] $) \cup\left(H_{0} \cap[\right.$ [n.s.d.] $)$. Since [p.s.i.] and [n.s.d.] are open, $H_{0} \cap$ [p.s.i.] and $H_{0} \cap$ [n.s.d.] are open subsets of $H_{0}$, in the relative topology. The above decomposition is then a disconnection of the connected set $H_{0}$, which gives the desired contradiction.

3. Linear equations of order $2 \mathbf{n}$. For the linear equation

$$
y^{(2 n)}-p(t) y=0
$$

we define the $(k, 2 n-k)$ focal point of $t=a$ as the smallest $b>a$ such that (3.1) has a nontrivial solution satisfying

$$
y(a)=\cdots=y^{(k-1)}(a)=0=y^{(k)}(b)=\cdots=y^{(2 n-1)}(b) .
$$

In case $n=2$ it is well known [2], [7] that the finiteness of the 2-2 focal point for all $a>0$ is equivalent to condition (0). Thus, denoting the $(k, 2 n-k)$ focal point by $\mu_{k, 2 n-k}(a)$, Ahmad's theorem can be restated as follows:

$$
(H) \Longleftrightarrow \mu_{2,2}(a)<\infty \text { for all } a<\infty .
$$

In this form a generalization of Ahmad's theorem is implicit in some recent work of Elias [2], and Lovelady [8]. In particular, one can combine the results of these authors to obtain the following.

THEOREM 3.1. If (3.1) is "oscillatory" in the sense that $\mu_{k, 2 n-k}(a)<$ $\infty$ for all $a<\infty$ and for all even integers $k, 2 \leqq k \leqq 2 n-2$, then (3.1) satisfies condition $(\mathrm{H})$.

Proof. If $y(t)$ is an eventually positive solution of (3.1) then there exists $c>0$ such that $y, y^{\prime}, \cdots, y^{(2 n-1)}$ are eventually one-signed. As shown in [1; Theorem 1], the largest integer $k$ such that $y^{(i)}>0$ on $[c, \infty)$ for all $i \leqq k$ is necessarily even, while for $j>k$ the $y^{(j)}$ have alternating signs. Thus, in order to establish $(\mathrm{H})$, it is sufficient to preclude trajectories which satisfy

$$
\begin{aligned}
& y^{(i)}>0 \text { on }[c, \infty) \text { for } 0 \leqq i \leqq k \\
& y^{(j)} y^{(j+1)}<0 \text { on }[c, \infty) \text { for } k \leqq j \leqq 2 n-1
\end{aligned}
$$

when $k=2,4, \cdots, 2 n-2$. However, specializing [2; Theorem 2] to 
the case of even order equations, it follows that (3.1) is $(k, 2 n-k)$ disfocal in $(a, \infty)$ if and only if it has a solution $y$ which satisfies

$$
\begin{aligned}
& y^{(i)}>0 \text { on }[a, \infty) \text { for } 0 \leqq i \leqq k \\
& (-1)^{j-k} y^{(i)}>0 \text { on }[a, \infty) \text { for } k \leqq j \leqq 2 n .
\end{aligned}
$$

Because of (3.2) we see that the finiteness of $\mu_{k, 2 n-k}(a)$ for even values of $k, 0<k<2 n$, and all $a<\infty$ sufficies to establish condition (H) for (3.1).

While we shall not make further use of this linear result, Theorem 3.1 does suggest that a focal point formulation of condition (0) is appropriate for generalizations of Ahmad's theorem. This point of view is pursued in connection with nonlinear fourth order equations in the following section.

4. Focal and asymptotic trajectories. In considering nonlinear equations of the form

$$
y^{i v}-f(t, y)=0 \quad 0 \leqq t<\infty
$$

we shall generalize the implication $(0) \Rightarrow(\mathrm{H})$ by showing that the existence of "asymptotic trajectories" which constitute violations of conditions $(\mathrm{H})$ is imcompatible with focal point properties which, in the linear case, are equivalent to condition (0). Writing (4.1) in the system form

$$
y^{\prime \prime}=x ; x^{\prime \prime}=f(t, y)
$$

it is clear that an eventually positive solution of (4.2) which is neither p.s.i. nor p.s.d. must eventually satisfy $y, y^{\prime}, x,-x^{\prime}$ positive, so that $y(t) \uparrow \infty$ and $x(t) \downarrow x_{0} \geqq 0$ as $t \rightarrow \infty$. In order to ensure that $x_{0}=0$ we assume that

$$
\begin{aligned}
& \text { (i) } f(t, y) \text { is monotonically increasing in } y \text { for } y \geqq 0 \\
& \text { (ii) for any } \varepsilon>0 \text { and } t_{0}>0, \int^{\infty} f\left(t, \varepsilon\left(t-t_{0}\right)^{2}\right) d t=\infty \text {. }
\end{aligned}
$$

LEMMA 4.1. If (4.3) is satisfied and $y(t)$ is an eventually positive solution of (4.1) which is neither p.s.i. nor p.s.d. then $y(t)$ satisfies

$$
y(t) \longrightarrow \infty \quad \text { and } \quad y^{\prime \prime}(t) \stackrel{\text { def }}{\equiv} x(t) \downarrow 0
$$

as $t \rightarrow \infty$.

Proof. If $y(t)$ is an eventually positive solution of (4.2), then there exists $t_{0}<\infty$ such that $y(t)>0$ and $y^{\prime \prime}(t)>x_{0}$ for $t \geqq t_{0}$. If, $x_{0}>0$ it follows that $y(t) \geqq \varepsilon\left(t-t_{0}\right)^{2}$ for some $\varepsilon>0$, and from (4.3) 
(i) and the second equation in (4.2) we have

$$
x^{\prime}(t) \geqq x^{\prime}\left(t_{0}\right)+\int_{t_{0}}^{t} f\left(s, \varepsilon\left(s-t_{0}\right)^{2}\right) d s .
$$

Therefore the divergence of the integral above precludes the existence of eventually positive solutions satisfying $x(t) \downarrow x_{0}$ for some $x_{0}>0$.

Analogous conclusions can be drawn for eventually negative solutions of (4.1), but we shall not consider this case explicitly. Rather we shall limit our attention to eventually positive solutions and assume (4.3) throughout, considering condition $(\mathrm{H})$ to be equivalent to the nonexistence of solutions of (4.1) satisfying (4.4).

Our nonlinear formulation of condition (0) is given in terms of the focal point $\eta\left(a ; x_{0}\right)$ defined as the smallest $b>a$ such that (4.1) has a nontrivial solution $y(t)$ satisfying

$$
y(a)=y^{\prime}(a)=0=y^{\prime \prime}(b)=y^{\prime \prime \prime}(b) ; y^{\prime \prime}(a)=x_{0} .
$$

A solution $y(t)$ of $(4.1)$ realizing $\eta\left(a ; x_{0}\right)$ can be interpreted as a trajectory in the $(x, y)$ plane tangent to the $x$-axis at $x=x_{0}, t=a$ and tangent to the $y$-axis at $t=\eta\left(a ; x_{0}\right)$. In case (4.1) is linear, $\eta$ does not depend on $x_{0}$. We then write $\eta\left(a ; x_{0}\right)=\tilde{\eta}(a)$, and existence of oscillatory solutions of (4.1) is well known to be equivalent to $\tilde{\eta}(a)<$ $\infty$ for all $a>0$. Defining a trajectory satisfying (4.4) as positively weakly increasing (p.w.i.), we seek to show that the existence of p.w.i. solution is inconsistent with condition ( 0 ) formulated as follows:

$$
\eta\left(a ; x_{0}\right)<\infty \text { for all } a \geqq 0 \text { and } x_{0}>0 \text {. }
$$

Our arguments will be given in a dynamical context wherein solutions of (4.2) satisfying initial conditions

$$
x(a)=x_{0}, y(a)=y_{0}, x^{\prime}(a)=x_{1}, y^{\prime}(a)=y_{1}
$$

are interpreted as trajectories in the $(x, y)$-plane. We also formulate initial conditions in terms of $x_{0}, y_{0}$,

$$
\theta_{0}=\operatorname{Arctan}\left[-\frac{y_{1}}{x_{1}}\right] ; v_{0}=\sqrt{x_{1}^{2}+y_{1}^{2}}
$$

and denote the corresponding trajectories by $\Gamma\left(x_{0}, y_{0}, \theta_{0}, v_{0} ; a\right)$. Then for each $\left(x_{0}, y_{0}\right) \in I^{+}$and $\theta_{0} \in[0,(\pi / 2))$ there exists a "critical velocity" $v_{c}\left(x_{0}, y_{0}, \theta_{0}\right)=\inf \left\{v \mid \Gamma\left(x_{0}, y_{0}, \theta_{0}, v\right)\right.$ enters the second quadrant\}. By continuous dependence on initial data, the corresponding critical trajectory $\Gamma_{c}\left(x_{0}, y_{0}, \theta_{0} ; a\right)=\Gamma\left(x_{0}, y_{0}, \theta_{0}, v_{c} ; a\right)$ is either focal, in the sense that it satisfies $x(\tau)=x^{\prime}(\tau)=0$ for some $\tau>a$, or else $\Gamma_{c}$ is asymptotic to the positive $y$-axis, corresponding to a p.w.i. solution of (4.1). We seek to show that if $\Gamma_{c}\left(x_{0}, 0,0 ; a\right)$ is focal for all $a \geqq 0$ 
and $x_{0}>0$, then there are no critical trajectories which are asymptotic to the positive $y$-axis-i.e., that $(0) \Rightarrow(H)$.

By way of preparation we establish an important result for non-homogeneous second order equations of the form

$$
\frac{d^{2} x}{d t^{2}}-q(t)=0
$$

where $q(t)>0$ on $(a, \infty)$. Given $x(a)=x_{0}>0$ we seek an initial velocity $x^{\prime}(a)=x_{1}<0$ such that the corresponding solution $x\left(t ; x_{0}, x_{1}\right)$ will satisfy $x(\tau)=x^{\prime}(\tau)=0$ for some $\tau \in(a, \infty]$. Such a critical $x_{1}$ is uniquely determined by the simultaneous solution of

$$
\begin{gathered}
x^{\prime}(t)=x_{1}+\int_{a}^{t} q(s) d s=0 \\
x(t)=x_{0}+x_{1} t+\int_{a}^{t} \int_{a}^{s} q(r) d r d s=0 .
\end{gathered}
$$

Our principal concern is the dependence of the critical arrival time $\tau$ on the function $q(t)$.

Lemma 4.2. Suppose $q_{i}(t)>0$ for $a<t<\infty$ and $i=1,2$. Let $x_{i}(t)$ be solutions of $\left(d^{2} x_{i} / d t^{2}\right)=q_{i}(t)$ satisfying $x_{i}(a)=x_{0}$ for $i=1,2$ and that $x_{i}^{\prime}(a)$ can be chosen so that $x_{i}\left(\tau_{i}\right)=x_{i}^{\prime}\left(\tau_{i}\right)=0$ for some $t_{i} \in(a, \infty] . \quad i=1,2$. If

$$
\int_{a}^{t}(s-a) q_{1}(s) d s \geqq \int_{a}^{t}(s-a) q_{2}(s) d s
$$

for all $t>a$, then $\tau_{1} \leqq \tau_{2}$. In particular, if $q_{1}(t) \geqq q_{2}(t)$ for all $t>a$, then $\tau_{1} \leqq \tau_{2}$.

Proof. From (4.3) we see that

$$
x_{i}^{\prime}(a)=-\int_{a}^{\tau_{i}} q_{i}(s) d s .
$$

Substituting into (4.4) yields

$$
x_{i}(t)=x_{0}^{-}(t-a) \int_{a}^{\tau_{i}} q_{i}(s) d s+\int_{a}^{t} \int_{a}^{s} q_{i}(r) d r d s .
$$

Interchanging the order of integration in the last term and setting $t=\tau_{i}$ yields

$$
0=x_{i}\left(\tau_{i}\right)=x_{0}^{-}\left(t-a_{i}\right) \int_{a}^{\tau_{i}} q_{i}(s) d s+\int_{a}^{\tau_{i}}\left(\tau_{i}-s\right) q_{i}(s) d s
$$

so that $\tau_{i}$ is determined by 


$$
x_{0}=\int_{a}^{\tau_{i}}(s-a) q_{i}(s) d s
$$

for $i=1,2$.

In the following application of Lemma 4.2 we use (4.3)(ii) and Lemma 4.1 to assure that the $X_{i}(a)$ can be chosen so that $X_{i}\left(\tau_{i}\right)=0$ for some $\tau_{i} \in(a, \infty]$.

LEMMA 4.3. Let $y_{1}(t)$ and $y_{2}(t)$ be critical solutions of (4.1) where $y_{2}(t)$ has a focal point $\eta\left(a ; x_{0}\right)<\infty$. If $y_{1}^{\prime \prime}(a)=y_{2}^{\prime \prime}(a)$ and $y_{1}(t) \geqq$ $y_{2}(t)$ for $a \leqq t \leqq \eta\left(a ; x_{0}\right)$, then $y_{1}(t)$ is not p.w.i.

Proof. Recalling that $x_{i}(t) \stackrel{\text { def }}{\equiv} y_{i}^{\prime \prime}(t)$, we note that the $x_{i}(t)$ are solutions of $x_{i}^{\prime \prime}=q_{i}(t)$, where $q_{i}(t)=f\left(t, y_{i}(t)\right)$ and $x_{1}(a)=x_{2}(a)=x_{0}$. From the monotonicity of $f$ and the assumption $y_{1} \geqq y_{2}$ it follows that $q_{1}(t) \geqq q_{2}(t)$ and that a critical $x_{1}^{\prime}(a)$ has been chosen so that $x_{1}(\tau)=x_{1}^{\prime}(\tau)=0$ for some $\tau \in\left(a, \eta\left(a ; x_{0}\right)\right.$. This means that the critical trajectory corresponding to $y_{2}(t)$ has a focal point in $\left(a, \eta\left(a ; x_{0}\right)\right)$ and therefore does not correspond to a p.w.i. solution.

At this point we are able to give a different linear proof of $(0) \Rightarrow(\mathrm{H})$. For if $y^{i v}-p(t) y=0$ has a p.w.i. solution $y_{1}(t)$ and if $\tilde{\eta}_{1}(a)<\infty$ for all $a$, then we can choose $t=a$ sufficiently large so that $y_{1}(t)>1$ for all $t \geqq a$. Letting $y_{2}(t)$ be the focal solution which realizes $\tilde{\eta}_{1}(\alpha)$, we can also choose $x_{2}(a) \stackrel{\text { def }}{\equiv} y_{2}^{\prime \prime}(a)$ sufficiently small so that $y_{2}(t)<1$ for $a \leqq t \leqq \tilde{\eta}_{1}(a)$. Then by Lemma 4.3 we obtain the desired contradiction. While this argument, as given, is valid only for the linear case, it can be modified to apply to certain nonlinear equations of the form (4.1).

Consider the Green's function

$$
\begin{aligned}
G(\tau ; t, s) & =\frac{\tau(s-a)(t-a)^{2}}{2}-\frac{\tau}{6}(t-a)^{3} \text { for } a<t<s<\tau \\
& =\frac{\tau(t-a)(s-a)^{2}}{2}-\frac{\tau}{6}(s-a)^{3} \text { for } a<s<t<\tau
\end{aligned}
$$

which enables us to write

$$
\begin{aligned}
& \frac{d^{4} y}{d t^{4}}-f(t, y(t)) \\
& y(a)=y^{\prime}(a)=0=y^{\prime \prime}(\tau)=y^{\prime \prime \prime}(\tau)
\end{aligned}
$$

in the form

$$
y(t)=\int_{a}^{\tau} G(\tau ; t, s) f(s, y(s)) d s
$$


Adopting the notation of [9] we let $K$ denote the cone of positive functions in the Banach space of bounded continuous functions on $[0, \infty)$. Using inequalities to indicate the partial ordering induced by $K$, we consider the positive operator $A_{\tau}$ defined by

$$
\left[A_{\tau} y\right](t)= \begin{cases}\int_{a}^{\tau} G(\tau ; t, s) f(r, y(s)) d s & a \leqq t \leqq \tau \\ 0 & t>\tau\end{cases}
$$

so that a focal point trajectory realizing $\eta\left(a ; x_{0}\right)$ can be identified with a solution of $A_{\tau} v=\mu_{\tau} v$ where

$$
v=y(t) ; \tau=\eta\left(a ; x_{0}\right) ; \mu_{\tau}=1 .
$$

(In order to remove the discontinuity at $t=\tau$ one should identify such a trajectory with

$$
\begin{aligned}
v(t) & =y(t) \text { for } & & a \leqq t \leqq \tau \\
& =y(2 \tau-t) & & \text { for } \tau \leqq t \leqq 2 \tau-a \\
& =0 & & \text { for } t>2 \tau-a
\end{aligned}
$$

but we shall not take further note of this technical detail.) In the linear case $f(t, y)=p(t) y$ we write

$$
\left[L_{\tau} y\right](t)= \begin{cases}\int_{a}^{\tau} G(t, s) p(s) y(s) d s & a \leqq t \leqq \tau \\ 0 & t>\tau\end{cases}
$$

so that a focal point trajectory realizing $\tilde{\eta}_{1}(a)$ can be identified with a solution of $L_{\tau} u=\lambda_{\tau} u$ where

$$
u=y(t) ; \tau=\tilde{\eta}_{1}(a) ; \lambda_{\tau}=1 .
$$

From the positivity and boundedness of the Green's function it follows that both $A_{\tau}$ and $L_{\tau}$ are completely continuous positive operators mapping $K$ into $K$ and that the focal point trajectories discussed above correspond to positive eigenvectors $u$ and $v$ of $L_{\tau}$ and $A_{\tau}$, respectively.

Lemma 4.4. Suppose $L_{\tau}$ and $A_{\tau}$ satisfy $L_{\tau} w \leqq A_{\tau} w$ for all $w \in$ $K$. If there exist nonzero elements $u, v \in K$ such that $L_{\tau} u=\lambda_{\tau} u$ and $A_{\tau} v=\mu_{\tau} v$, then $\lambda_{\tau} \leqq \mu_{\tau}$.

Proof. Since $L_{\tau}$ is linear, $u$ may be multiplied by an arbitrary positive constant, so that we may assume without loss of generality that $u-v \in K-\{0\}$. Assuming $\lambda_{\tau}>\mu_{\tau}$ we write

$$
L_{\tau}(u-v)+\left(L_{\tau}-A_{\tau}\right) v=\lambda_{\tau}(u-v)+\left(\lambda_{\tau}-\mu\right) v
$$


and conclude that

$$
L_{\tau}(u-v)-\lambda_{\tau}(u-v) \in K-\{0\}
$$

for the positive element $u-v$. However this contradicts the assumption that $\lambda_{\tau}$ is the spectral radius of $L_{\tau}$ (see [9; Theorem 2.5]).

THEOREM 4.5. Suppose there exists a function $p(t)$ which is continuous and positive on $[0, \infty)$, satisfies

$$
f(t, y) \geqq p(t) y \quad \text { for } \quad(t, y) \in[0, \infty) \times(0, \infty)
$$

and such that $y^{i v}-p(t) y$ has $\tilde{\eta}_{1}(a)<\infty$ for all $a>0$. If (4.1) satisfies $\eta_{1}\left(a ; x_{0}\right)<\infty$ for all $a \geqq 0$ and $x_{0}>0$ then (4.1) does not have any p.w.i. solutions.

Proof. Suppose to the contrary that (4.1) has a p.w.i. solution $y_{1}(t)$ satisfying

$$
y_{1}(t)>1 \text { for all } t \geqq a ; \lim _{t \rightarrow \infty} y_{1}(t)=\infty ; \lim _{t \rightarrow \infty} y_{1}^{\prime \prime}(t)=0 \text {. }
$$

If $y_{2}\left(t ; x_{0}\right)$ is the solution of (4.1) realizing $\eta\left(a ; x_{0}\right)$ and $v \in K$ is identified with $y_{2}\left(t ; x_{0}\right)$, then $A_{\tau} v=\mu_{\tau} v$, with $\tau\left(a ; x_{0}\right)=\eta_{1}\left(a ; x_{0}\right)$ and $\mu_{\tau}=1$. Making use of the fact that $L_{\tau}$ is a compact positive linear operator, we note that

$$
L_{\tau} u=\lambda_{\tau} u
$$

has an essentially unique solution $u \in K$ corresponding to $\lambda_{\tau} \leqq \mu_{\tau}=$ 1. By the classical variational theory, $\lambda_{\tau}$ is a monotonically increasing function of $\tau$, so that there exists $\widetilde{\tau} \geqq \tau$ such that

$$
L_{\tau}^{\sim} u=u
$$

has a nontrivial solution in $K$ corresponding to the solution of $y^{i v}-$ $p(t) y=0$ realizing $\tilde{\tau}_{1}(a)$, and $\bar{\eta}_{1}=1$. This shows that $\eta_{1}\left(a ; x_{0}\right) \leqq$ $\tilde{\eta}_{1}(a)$ for all $x_{0}>0$ and, as a consequence, that $y_{2}\left(t ; x_{0}\right) \leqq\left(x_{0} / 2\right)(\tau-a)^{2}$ for $a \leqq t \leqq \eta_{1}\left(a ; x_{0}\right)$. Choosing $x_{0}$ sufficiently small, we obtain $y_{2}\left(t ; x_{0}\right) \leqq$ $y_{1}(t)$ for $a \leqq t \leqq \eta_{1}\left(a ; x_{0}\right)$ and apply Lemma 4.3 to obtain the desired contradiction.

\section{REFERENCES}

1. S. Ahmad, On the oscillation of a class of linear fourth order differential equations, Pacific J. Math., 34 (1970), 289-299.

2. U. Elias, Necessary conditions and sufficient cnoditions for disfocality and disconjugacy of a differential equation, Pacific J. Math., 81 (1979), 379-397.

3. G. J. Etgen and W. E. Taylor, Jr., On the oscillation of a class of fourth order differential equations, Rocky Mountain. J. Math., 6 (1976), 71-84. 
4. E. Hille, Lectures on Ordinary Differential Equations, Addison Wesley, 1969.

5. M. A. Krasnosel'skii, Positive Solutions of Operator Equations, Noordhoff, Groningen, Netherlands, 1964.

6. K. Kreith, Nonlinear differential equations with monotone solutions, Pacific J. Math., 81 (1979), 101-111.

7. W. Leighton and Z. Nehari, On the oscillation of solutions of self-adjoint linear differential equations of the fourth order, Trans. Amer. Math. Soc., 89 (1958), 325-377.

8. D. L. Lovelady, An asymptotic analysis of an even order linear differential equation, Funkcialaj Ekvacioj, 19 (1976), 133-138.

9. K. Schmitt and H. L. Smith, Positive solutions and conjugate points for systems of differential equations, J. Nonlinear Finl. Anal., to appear.

Received October 9, 1978 and in revised form January 4, 1980.

UNIVERSITY OF CALIFORNIA

Davis, CA 95616 



\section{PACIFIC JOURNAL OF MATHEMATICS}

\section{EDITORS}

DONALD BABBITT (Managing Editor)

University of California

Los Angeles, California 90024

HUGO ROSSI

University of Utah

Salt Lake City, UT 84112

C. C. MOORE and ARTHur AGUS

University of California

Berkeley, CA 94720
J. DUGUNDJI

Department of Mathematics

University of Southern California

Los Angeles, California 90007

R. FINN and J. MILGRAM

Stanford University

Stanford, California 94305

\section{ASSOCIATE EDITORS}
R. Arnes
E. F. BECKENBACH
B. H. NeumanN
F. WOLF
K. YösHIDA

\section{SUPPORTING INSTITUTIONS}

UNIVERSITY OF ARIZONA

UNIVERSITY OF BRITISH COLUMBIA

CALIFORNIA INSTITUTE OF TECHNOLOGY

UNIVERSITY OF CALIFORNIA

MONTANA STATE UNIVERSITY

UNIVERSITY OF NEVADA, RENO

NEW MEXICO STATE UNIVERSITY

OREGON STATE UNIVERSITY
UNIVERSITY OF OREGON

UNIVERSITY OF SOUTHERN CALIFORNIA

STANFORD UNIVERSITY

UNIVERSITY OF HAWAII

UNIVERSITY OF TOKYO

UNIVERSITY OF UTAH

WASHINGTON STATE UNIVERSITY

UNIVERSITY OF WASHINGTON 


\section{Pacific Journal of Mathematics}

Vol. 102, No. $1 \quad$ January, 1982

S. Agou, Degré minimum des polynômes $f\left(\sum_{i=0}^{m} a_{i} X^{p^{r i}}\right)$ sur les corps finis

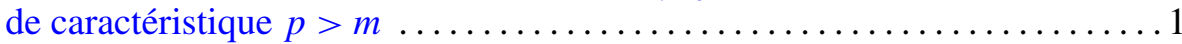

Chi Cheng Chen, On the image of the generalized Gauss map of a complete

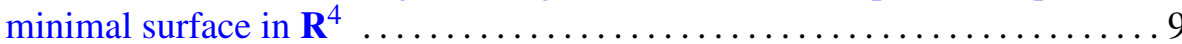

Thomas Curtis Craven and George Leslie Csordas, On the number of real

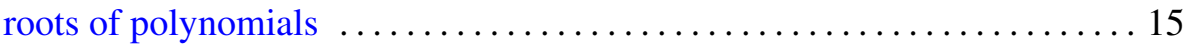

Allan L. Edelson and Kurt Kreith, Nonlinear relationships between oscillation and asymptotic behavior ....................... 29

B. Felzenszwalb and Antonio Giambruno, A commutativity theorem for

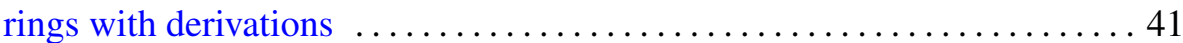

Richard Elam Heisey, Manifolds modelled on the direct limit of lines . . . . . 47

Steve J. Kaplan, Twisting to algebraically slice knots $\ldots \ldots \ldots \ldots \ldots \ldots 5$

Jeffrey C. Lagarias, Best simultaneous Diophantine approximations. II.

Behavior of consecutive best approximations $\ldots \ldots \ldots \ldots \ldots \ldots \ldots 61$

Masahiko Miyamoto, An affirmative answer to Glauberman's conjecture . . . 889

Thomas Bourque Muenzenberger, Raymond Earl Smithson and L. E.

Ward, Characterizations of arboroids and dendritic spaces ........... 107

William Leslie Pardon, The exact sequence of a localization for Witt

groups. II. Numerical invariants of odd-dimensional surgery

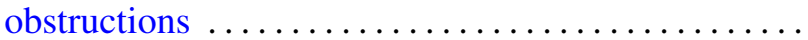

Bruce Eli Sagan, Bijective proofs of certain vector partition identities

Kichi-Suke Saito, Automorphisms and nonselfadjoint crossed products ...

John Joseph Sarraille, Module finiteness of low-dimensional PI rings ...

Gary Roy Spoar, Differentiable curves of cyclic order four . .

William Charles Waterhouse, Automorphisms of quotients of $\Pi \mathrm{GL}\left(n_{i}\right)$

Leslie Wilson, Mapgerms infinitely determined with respect to right-left equivalence

Rahman Mahmoud Younis, Interpolation in strongly logmodular

algebras 